\title{
Benchmarking Correlated Methods for Frequency-Dependent Polarizabilities: Aromatic Molecules with the CC3, CCSD, CC2, SOPPA, SOPPA(CC2), and SOPPA(CCSD) Methods
}

Jørgensen, Maria W; Faber, Rasmus; Ligabue, Andrea; Sauer, Stephan P. A.

Published in:

Journal of Chemical Theory and Computation

Link to article, DOI:

$10.1021 /$ acs.jctc. 9 b01300

Publication date:

2020

Document Version

Publisher's PDF, also known as Version of record

Link back to DTU Orbit

Citation $(A P A)$ :

Jørgensen, M. W., Faber, R., Ligabue, A., \& Sauer, S. P. A. (2020). Benchmarking Correlated Methods for Frequency-Dependent Polarizabilities: Aromatic Molecules with the CC3, CCSD, CC2, SOPPA, SOPPA(CC2), and SOPPA(CCSD) Methods. Journal of Chemical Theory and Computation, 16(5), 3006-3018. https://doi.org/10.1021/acs.jctc.9b01300

\section{General rights}

Copyright and moral rights for the publications made accessible in the public portal are retained by the authors and/or other copyright owners and it is a condition of accessing publications that users recognise and abide by the legal requirements associated with these rights.

- Users may download and print one copy of any publication from the public portal for the purpose of private study or research.

- You may not further distribute the material or use it for any profit-making activity or commercial gain

- You may freely distribute the URL identifying the publication in the public portal 


\title{
Benchmarking Correlated Methods for Frequency-Dependent Polarizabilities: Aromatic Molecules with the CC3, CCSD, CC2, SOPPA, SOPPA(CC2), and SOPPA(CCSD) Methods
}

\author{
Maria W. Jørgensen, Rasmus Faber, Andrea Ligabue, and Stephan P. A. Sauer* \\ Cite This: https://dx.doi.org/10.1021/acs.jctc.9b01300 \\ Read Online
}

\section{ACCESS}

Џlll Metrics \& More

回国 Article Recommendations

Supporting Information

ABSTRACT: A benchmark study of several correlated secondorder methods for frequency-dependent polarizabilities has been carried out. For the benchmark, a set of 14 (hetero)aromatic medium-sized molecules has been chosen. For the first time, CC3 polarizabilities are reported for these molecules using Sadlej's polarized triple- $\zeta$ basis set, and for a subset of these molecules the polarizabilities were obtained at the CC3 level also with the larger aug-cc-pVTZ basis set. These CC 3 values are used as the reference values for benchmarking the second-order methods: SOPPA, SOPPA(CC2), SOPPA(CCSD), CC2, as well as CCSD. The influence of different basis sets, aug-cc-pVDZ, aug-cc-pVTZ, aug-

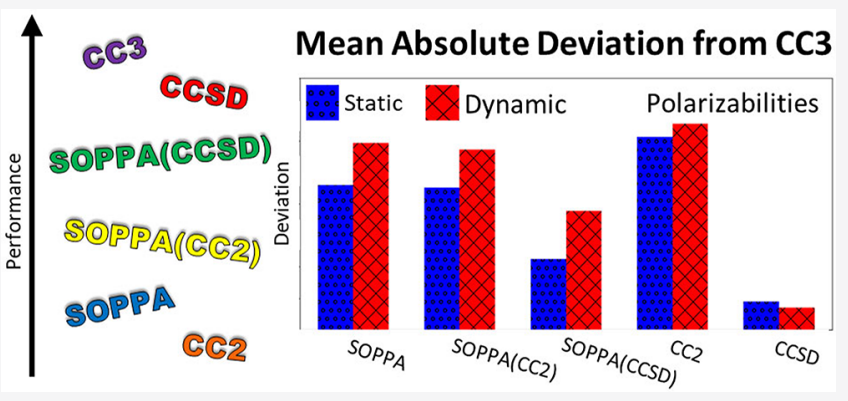
cc-pVQZ, d-aug-cc-pVTZ, and Sadlej's polarized triple- $\zeta$ basis set, on static and frequency-dependent polarizabilities was investigated for the full set of molecules at the SOPPA level. It was found that the choice of basis set had a somewhat greater influence on the frequency-dependent polarizabilities than on the static polarizabilities, but all effects were small. The aug-cc-pVTZ basis set performed adequately for both static and frequency-dependent polarizabilities, having an insignificant offset from the values obtained with the larger d-aug-cc-pVTZ and aug-cc-pVQZ basis sets. Comparing the second-order methods, SOPPA, SOPPA(CC2), SOPPA(CCSD), CC2, as well as CCSD, to the CC3 reference values, it was found that the best performing method was CCSD, as expected. The SOPPA method, on the contrary, outperformed the CC2 method, suggesting the use of SOPPA rather than CC2 for polarizabilities, at least for these kinds of molecules. The SOPPA results were found to improve further when the Møller-Plesset correlation coefficients in the wave function were replaced by coupled-cluster amplitudes in the SOPPA(CC2) and SOPPA(CCSD) methods. Finally, a comparison was made for a small subset of the molecules between experimental data and calculated polarizabilities. It shows that, for this set of molecules, the trend in the performance of the different second-order methods does not depend on whether the reference values are calculated CC3 values or experimental values.

\section{INTRODUCTION}

The interaction of molecules with light causes a perturbation of the electrons in the molecule. Such a polarization plays an important role in molecular interactions and influences the way in which light scatters from a molecule. ${ }^{1}$ At high intensities, caused by lasers, the molecular response to the field yields properties such as two-photon absorption, self-focusing, highharmonic generation, and more. These nonlinear optical effects are of great importance, ${ }^{2}$ and there is a need for efficient and accurate methods to calculate these properties. A natural prerequisite for such methods is that they should first perform well for linear optical properties. Linear optics, as the name suggests, possesses the advantage that at a low intensity of laser light, the response of the molecule is linear in the field. Such a property could be the electronic dipole polarizability, which is the focus of this study. The polarizability or, more precisely, its gradients with respect to normal modes, determines the intensities of vibrational Raman transitions. ${ }^{3}$ The electric dipole polarizability tensor and its geometric derivatives also play a substantial role in electron energy-loss spectra (EELS)..$^{4-6}$ The electronic polarizability has also been found to affect the mechanism in multiphoton absorption. ${ }^{7}$ Calculated polarizabilities can thus be used to verify a variety of different experimental data and can also be used for the prediction of polarizabilities of molecules for which experimental data are not yet available. However, this is only possible if the calculations have a similar accuracy as the experiments. As the accuracy of the experimental methods keeps improving, there has been a great interest in developing more advanced theories while still keeping the computational cost low.

Received: December 31, 2019

Published: April 17, 2020 
The quest for well-performing methods has led to the benchmarking of many computational methods for several molecular properties in recent literature. Benchmark studies are of great importance and will continue to be so, as long as new methods are developed. Comparing medium-cost methods to higher cost methods can verify the performance of medium-cost methods, which can then be used with more confidence in large-scale applications. Benchmark studies also provide reference values for future investigations. The calculation of electronic excitation energies has most extensively been investigated with the benchmark set of Thiel and coworkers, ${ }^{8-10}$ leading to many following studies. Benchmark studies have also been published for linear response properties like NMR coupling constants. ${ }^{11-14}$

Many studies of polarizabilities have been carried out using response theory methods. As coupled-cluster theory has become a promising theory during the last several decades, many studies have been carried out at this level of theory. The hierarchical improvement of coupled-cluster methods has, for example, been demonstrated for polarizabilities by a study of Larsen et al., ${ }^{15}$ showing that the performance improves in the series CCS, CC2, CCSD, and CC3. Christiansen et al. ${ }^{16}$ showed that this systematic improvement also extends to CCSDT. Several approximate coupled-cluster methods and variants, for example, the Brueckner coupled-cluster method, ${ }^{17}$ have been developed from this theory. Furthermore, there has been an increase in interest in second-order methods like the second-order polarization propagator approximation (SOPPA), ${ }^{18,19} \mathrm{CC} 2,{ }^{20}$ or $\mathrm{ADC}(2),{ }^{21,22}$ as higher-order methods are, for larger systems, still greatly limited by computing power. Also, the usage of coupled-cluster groundstate amplitudes in other theories such as SOPPA and ADC theory has been proposed, leading to intermediate models like SOPPA(CC2), ${ }^{12}$ SOPPA(CCSD), ${ }^{23,24}$ or CCD-ADC(2) and $\operatorname{CCSD}-\operatorname{ADC}(2){ }^{25,26}$

A study by Packer et al. ${ }^{27}$ showed that SOPPA performs better than time-dependent Hartree-Fock (TDHF) for frequency-dependent polarizabilities. This was also the case in the study of static polarizabilities by Dalskov and Sauer, ${ }^{28}$ where they found an improvement of the methods in the series TDHF $<$ SOPPA < SOPPA $($ CCSD $)<$ CCSD. The observation that SOPPA is out-performed by SOPPA(CCSD) for polarizabilities has also been made in several other studies $^{23,29-32}$ on small molecules. The improvement of the SOPPA method by using the coupled-cluster amplitudes inspired the study by Hodecker et al., ${ }^{25}$ where the coupledcluster amplitudes were incorporated in the $\operatorname{ADC}(2)$ method and tested primarily for excitation energies. No significant effect was observed for most cases of smaller molecules, but the performance for the difficult case of ozone was improved in the CCD-ADC(2) method. Similar to a previous SOPPA(CCSD) study, ${ }^{24}$ no real effect was found, in general, for singlet excited states of aromatic organic molecules, but an improvement was observed for triplet excited states. The study was expanded ${ }^{26}$ to also test the larger coupled-cluster version of the method, CCSD-ADC(2). In this study, the methods were tested for polarizabilities of aromatic organic molecules because the cases of, for example, benzene and pyridine had been shown to be difficult for the ADC method. The same tendency as for the SOPPA method was shown, meaning the higher the order of the coupled-cluster amplitudes, the more improved the method becomes. Another approach to obtain results of high precision for larger molecules is to lower the cost of demanding preexisting methods. This was, for example, done by Pedersen et al. in a study ${ }^{33}$ where the implementation of the CC2 method was optimized based on a Cholesky decomposition of the two-electron integrals. Alternatively, the resolution of the identity approximation ${ }^{34,35}$ can significantly reduce the CPU time of CC2 calculations.

For small molecules, there are many calculations of static or dynamic polarizabilities using various forms of coupled-cluster or Møller-Plesset perturbation theory, and it would go to far for this Article to mention more than a few. ${ }^{22,28,36-43}$ We are aware of only a few high-level calculations on larger molecules. Unsurprisingly, benzene appears to be a very popular molecule for testing new methods. ${ }^{22,26,33,44-47}$ Sadlej's polarized triple- $\zeta$ basis $\operatorname{set}^{48,49}$ as well as various correlation consistent basis sets ${ }^{50-52}$ have been employed in these studies. A few previous papers are worth mentioning explicitly here. Pedersen et al. ${ }^{33}$ carried out an extensive basis set study of the static polarizability of benzene at the CC2 level going up to even an aug-cc-pV7Z basis set. Coriani et al., ${ }^{47}$ on the contrary, reported a CC3 value for the static polarizability of benzene calculated with the aug-cc-pVDZ basis set, and Fransson et $\mathrm{al}^{22}$ reported an $\operatorname{ADC}(3 / 2)$ value for the static polarizability of benzene using a triple- $\zeta$ basis set. For furan, static and frequency-dependent polarizabilities have been reported at the CC2 and CCSD levels with the aug-cc-pVDZ basis set. ${ }^{53}$ Static polarizabilities for pyridine and naphthalene were very recently reported using various versions of ADC methods and Sadlej's basis set, $^{26}$ as previously mentioned. Finally, the static polarizabilities of small and large $[4 n+2]$ annulenes were studied at the CC2/aug-cc-pVDZ level of theory. ${ }^{46,54}$ There are only two studies, of which we are aware, where for a larger set of medium-sized (hetero)aromatic molecules CCSD results were reported. The first is the 2014 study of Hickey and Rowley, ${ }^{37}$ who used both Sadlej's polarized triple- $\zeta$ and the aug-cc-pVTZ basis sets on furan, thiophene, pyrrole, pyrazole, imidazole, benzene, pyridine, toluene, fluorobenzen, chlorobenzene, phenol, and imidazole. The other study is by Skrzyński et al., ${ }^{55}$ who used Sadlej's basis set for CCSD as well as CC2 for fluorene, carbazole, and dibenzofuran. On the contrary, the focus for polarizabilities has not only been on wave-function methods but also been on the often faster density functional theory (DFT) methods, which has led to several benchmark studies through the years. ${ }^{56-58}$ Hickey and Rowley ${ }^{37}$ compared both wave-function and DFT methods in their calculation of dipole moments and polarizabilities for a variety of molecules, from smaller diatomic molecules to (hetero)aromatic systems like those in this study. It was found that CCSD performed similarly to some of the DFT functionals when comparing to experimental values. However, when comparing to CCSD results, it was clear that the secondorder method MP2 did perform better than the DFT methods.

Despite all of these studies of polarizabilities with different methods, until now, no direct comparison of SOPPA and coupled-cluster methods has been carried out for polarizabilities of larger molecules. This study thus focuses on the benchmarking of second-order methods such as the coupledcluster method CC2 and SOPPA in addition to the full coupled-cluster single- and double-model CCSD for polarizabilities of (hetero)aromatic molecules. The CCSD model contains both the single- and double-excitation operators, whereas in the CC2 model, the equations for the double amplitudes are approximated based on Møller-Plesset perturbation theory. Similarly in SOPPA, the double-excitation 
part of the molecular Hessian is evaluated only to the zeroth order. Furthermore, two mixed methods have been included, the SOPPA(CC2) and SOPPA(CCSD) methods. These methods draw on the fact that the coupled-cluster wave function is a better description of the electronic ground-state wave function than the second-order Møller-Plesset theory. The reference state in the expression for the polarization propagator is thus approximated by a linearized coupledcluster wave function. This has essentially no influence on the SOPPA equations but improves the description of the electron correlation due to an improved wave function.

For the benchmark, the second-order methods should be compared to a method of adequate performance. Thus, in this study, the CC3 method ${ }^{59}$ has been used. Optimally, the full CCSDT method ${ }^{60}$ should have been used, but this method, as it now includes the full equations for the triple amplitudes, is simply too expensive for the size of molecules studied in this work. The CC3 method, on the contrary, takes advantage of the same approximation as the CC2 method for the doubleamplitude equations by approximating the equation for the triple amplitude. As expected, the CC3 method has been shown to perform better than CCSD for frequency-dependent polarizabilities. $^{61}$

\section{COMPUTATIONAL DETAILS}

The set of molecules in this study consists of 14 aromatic medium-sized molecules, whose structures can be seen in Figure 1. Geometries optimized at the MP2/6-31G(d) level for<smiles>c1ccccc1</smiles>

Benzene<smiles>c1ccncc1</smiles><smiles>c1cncnc1</smiles>

Pyridine

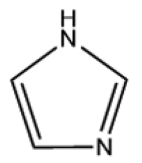

Imidazole

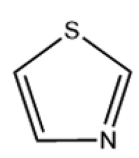

Thiazole<smiles>c1ccnnc1</smiles><smiles>Oc1ccccc1</smiles>

Phenol

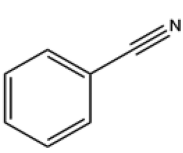

Benzonitrile

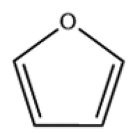

Furan

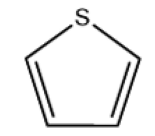

Thiophene

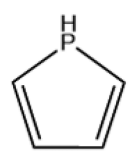

Phosphole

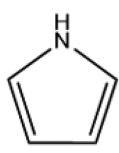

Pyrrole
Figure 1. Structures and names of the molecules used in this study.

benzene, furan, pyrrole, imidazole, pyridine, pyrimidine, pyrazine, and pyridazine were taken from the literature. ${ }^{9}$ The geometries of the remaining molecules were optimized at the same level of theory using the Gaussian ${ }^{62}$ program. The level of optimization was thus kept the same as in the Thiel benchmark set for excitation energies ${ }^{8-10}$ for the current polarizability benchmark to function as an extension. For the study of the basis set dependence of the polarizabilities, Dunning's aug-cc-pVDZ, aug-cc-pVTZ, aug-cc-pVQZ, and daug-cc-pVTZ basis sets ${ }^{50-52}$ were used as well as Sadlej's polarized triple- $\zeta$ basis set. ${ }^{48,49}$

For the method benchmarking part of this study, the aug-ccpVTZ and Sadlej basis sets were used. The polarizabilities in this study, calculated with the SOPPA, SOPPA(CC2),
SOPPA(CCSD), CC2, and CCSD methods, were obtained with the DALTON program. ${ }^{63}$ The reference polarizabilities, calculated with the CC3 method with the Sadlej basis set, were also obtained with DALTON, whereas the CFOUR program ${ }^{64}$ was used for the reference values with the larger aug-cc-pVTZ basis set. The SOPPA-based methods are, by derivation, unrelaxed methods, but all coupled-cluster results were also obtained with the unrelaxed approach to ensure the correct pole structure, which is especially important for the dynamic polarizabilities. The benchmarking is carried out for both static and dynamic polarizabilities. The frequencies used in this study were $632.8 \mathrm{~nm}(0.072003 \mathrm{au})$, HeNe laser, and $488.8 \mathrm{~nm}$ $(0.093215 \mathrm{au})$. They were chosen because they were frequently found to be used in experiments in previous literature. All polarizabilities reported in this work are isotropic polarizabilities, that is, one-third of the trace of polarizability tensor.

\section{RESULTS AND DISCUSSION}

In Section 3.1 there will be a discussion of the performance of the different basis sets studied in this work to find the optimal basis set for further usage. Section 3.2 will be a discussion of finding appropriate reference values to use for benchmarking of the second-order methods and how to obtain them in a feasible way. In Section 3.3, the different second-order methods will be compared with the coupled-cluster method CC3 to test their accuracy. At last, in Section 3.4, there will be a comparison between experimental and calculated polarizabilities for a smaller set of molecules.

3.1. Basis Set Effects. For any calculation, a sufficient, well-performing basis set is of high importance for the accuracy of the results. Previously, it has been shown that the accuracy of polarizabilities is highly dependent on the basis set. ${ }^{65,66}$ It was proposed that a triple- $\zeta$ basis set contains enough basis functions to perform sufficiently. Because the polarizability is the property in focus, diffuse functions should also be included to better describe the long-range behavior. Second, it was shown that the neglect of diffuse functions, which better describe the electron correlation, leads to significant errors when calculating electronic properties. ${ }^{37}$ It was also found that the aug-cc-pVDZ, aug-cc-pVTZ, and Sadlej's cc-pVTZ basis sets gave similar results, with the aug-cc-pVTZ basis set performing best. Another study ${ }^{16}$ states that although the diffuse functions are critical for polarizabilities, more than one set is not needed, and one should instead go to higher cardinal numbers. Thus, in the present study, the aug-cc-pVTZ basis set is tested against both the smaller aug-cc-pVDZ basis set and the larger aug-cc-pVQZ basis set to see how much is gained or lost in accuracy by using the triple- $\zeta$ basis set over the larger or smaller ones. Furthermore, the accuracy gained by employing even more diffuse functions, using the d-aug-cc-pVTZ basis set, is tested. Finally, also, a basis set specialized for polarizabilities, Sadlej's polarized triple- $\zeta$ basis set, is tested against the aug-ccpVTZ basis set. In this study, the test of the basis sets is separately carried out for static and dynamic polarizabilities, and both cases are calculated with the SOPPA method. All of the results can be found in Tables S1 and S2.

For the separate static and dynamic cases, linear regressions between the SOPPA/aug-cc-pVTZ results and the SOPPA polarizabilities obtained with the other basis sets were generated. Because all basis sets exhibit a good correlation with the aug-cc-pVTZ basis set, not much can be seen from the plots, which can be found in Figures S1 and S2. On the 
Table 1. Parameters in the Linear Regression $y=a x+b$ of Static and Dynamic Polarizabilities (in au), Calculated with the Given Basis Set $(y)$ versus the Values Obtained with the aug-cc-pVTZ Basis Set $(x)^{a}$

\begin{tabular}{|c|c|c|c|c|c|c|}
\hline \multirow[b]{2}{*}{ basis set } & \multicolumn{3}{|c|}{ static } & \multicolumn{3}{|c|}{ dynamic } \\
\hline & $a$ & $b$ & $R^{2}$ & $a$ & $b$ & $R^{2}$ \\
\hline aug-cc-pVDZ & 0.9970 & 0.4682 & 0.9999 & 0.9960 & 0.5387 & 0.9999 \\
\hline aug-cc-pVQZ & 0.9976 & -0.1494 & 1.0000 & 0.9976 & -0.1616 & 1.0000 \\
\hline d-aug-cc-pVTZ & 1.0001 & 0.1005 & 1.0000 & 0.9998 & 0.1424 & 1.0000 \\
\hline Sadlej & 1.0018 & 0.4905 & 0.9998 & 1.0012 & 0.5916 & 0.9997 \\
\hline
\end{tabular}

Table 2. Mean Deviations (in au) of the Static and Dynamic Polarizabilities Calculated with the Different Basis Sets from the aug-cc-pVTZ Results ${ }^{a}$

\begin{tabular}{|c|c|c|c|c|c|c|}
\hline \multirow[b]{2}{*}{ basis set } & \multicolumn{3}{|c|}{ static } & \multicolumn{3}{|c|}{ dynamic } \\
\hline & mean & abs. mean & std. dev. & mean & abs. mean & std. dev. \\
\hline aug-cc-pVDZ & 0.27 & 0.27 & 0.13 & 0.27 & 0.27 & 0.15 \\
\hline aug-cc-pVQZ & -0.31 & 0.31 & 0.04 & -0.32 & 0.32 & 0.05 \\
\hline d-aug-cc-pVTZ & 0.11 & 0.11 & 0.04 & 0.13 & 0.13 & 0.05 \\
\hline Sadlej & 0.60 & 0.60 & 0.17 & 0.67 & 0.67 & 0.23 \\
\hline
\end{tabular}

${ }^{a}$ Dynamic polarizabilities for the frequencies 0.072003 and 0.93215 au.

contrary, the parameters from the linear regressions, which can be found in Table 1, show the differences between the basis sets more clearly. The parameters for the static case reveal that all correlations are close to perfect and have only small offsets compared with the size of the polarizabilities, that is, at most $1 \%$. The slope for the correlation between the aug-cc-pVDZ and aug-cc-pVTZ basis sets is the one most deviating from 1 , and the offset is rather large. This means that the accuracy of the results will be less if the double- $\zeta$ basis set is used instead of the triple- $\zeta$ basis set. For the correlation between the aug-ccpVQZ and aug-cc-pVTZ basis sets, the $R^{2}$ value is perfect, the slope is very close to 1 , and the intercept is close to 0 , that is, slightly lower than 0 . This shows that the gain in accuracy by using the quadruple- $\zeta$ basis set instead of the triple- $\zeta$ basis set is very small. Taking the longer computational time into consideration, it is thus well justified to use the triple- $\zeta$ basis set. The inclusion of even more diffuse functions in the d-augcc-pVTZ basis set is seen to have an insignificant effect on the results, as the correlation between the basis sets is almost perfect. Therefore, there is no need to use the extra computational time on the d-aug-cc-pVTZ basis set because there is no improvement in the accuracy. Sadlej's basis set is seen to have the lowest $R^{2}$ value, and the largest offset for the correlation with the aug-cc-pVTZ basis set. However, because the slope deviates only 0.0018 from 1 , the loss of accuracy would be little if the offset was corrected for. From this, it can be concluded that because all basis sets perform similarly, for the static polarizabilities of such systems, any of the basis sets would give reliable results. Using the aug-cc-pVTZ instead of the larger basis sets will have an insignificant effect on the accuracy of the results.

For the dynamic polarizabilities, the changes in the basis sets are seen to have a slightly larger effect, which may not be so obvious from the plots in Figure S2 but can be observed by looking at the values in Table 1 . The aug-cc-pVQZ basis set is again found to perform almost equally to the smaller aug-ccpVTZ basis set, with a perfect $R^{2}$ value, a small offset, and a slope close to 1 . It is thus more than justified to use the aug-ccpVTZ instead of the larger basis set because there is no real gain in accuracy using the larger one. The lowered computa- tional time further justifies this. In the same way, the effect of adding more diffuse functions to the aug-cc-pVTZ, making it double-augmented, is again also seen to give only a negligible gain of accuracy. There is thus no need to use the extra computational time necessary for using the d-aug-cc-pVTZ in place of the aug-cc-pVTZ basis set. As for the static case, Sadlej's polarized triple- $\zeta$ basis set is again found to have a lower $R^{2}$ value than the aug-cc-pVDZ basis set and a larger offset for the correlation with the results of the aug-cc-pVTZ basis set. However, the slope is again closer to 1 , meaning that the offset could be corrected, and then this basis set would perform comparably to the aug-cc-pVTZ basis set.

To look further into the performance of the different basis sets, mean and absolute mean deviations from the aug-ccpVTZ results were calculated for each basis set. They can be found in Table 2, again separated for the static and dynamic cases. The values prove that all of the basis sets perform rather close to the aug-cc-pVTZ basis set because the deviations are small compared with the size of the polarizabilities. Although both d-aug-cc-pVTZ and aug-cc-pVQZ consist of more basis functions than aug-cc-pVTZ, their results are again seen to differ only insignificantly from the aug-cc-pVTZ results. It can be seen for both the static and dynamic cases that the aug-ccpVDZ results deviate less and have a smaller spread in the deviation than the Sadlej's basis set results. This might seem unexpected, considering that Sadlej's basis set contains more basis functions than aug-cc-pVDZ. However, Sadlej's basis set has been found to be affected by the near-linear dependence of the response properties for larger molecules. ${ }^{67}$ Hence, the molecules in this study might approach a large enough size for this effect to be become relevant.

Overall, aug-cc-pVTZ should give results of sufficient precision for both the static and frequency-dependent cases because there is not a great gain in accuracy using the larger basis sets. There is, on the contrary, a small gain in using the aug-cc-pVTZ over the aug-cc-pVDZ and Sadlej's basis sets. For this reason, the aug-cc-pVTZ basis set is used in this study. The smaller basis sets can be adequate if a quicker estimate of polarizabilities is needed. 
Table 3. CC3 Static and Dynamic Isotropic Polarizabilities (in au) Obtained with Sadlej's Basis Set and the aug-cc-pVTZ Basis Set, aT, and by Estimating the aug-cc-pVTZ Values, (aT), from the Correlation in Equation 1

\begin{tabular}{|c|c|c|c|c|c|c|c|c|c|}
\hline \multirow[b]{3}{*}{ molecule } & \multicolumn{9}{|c|}{ frequency $(\mathrm{au})$} \\
\hline & \multicolumn{3}{|c|}{0.000000} & \multicolumn{3}{|c|}{0.072003} & \multicolumn{3}{|c|}{0.093215} \\
\hline & Sadlej & $\mathrm{aT}^{a}$ & $(\mathrm{aT})^{a}$ & Sadlej & $\mathrm{aT}^{a}$ & $(\mathrm{aT})^{a}$ & Sadlej & $\mathrm{aT}^{a}$ & $(\mathrm{aT})^{a}$ \\
\hline benzene & 68.76 & 68.49 & 68.28 & 71.16 & 70.86 & 70.66 & 72.96 & 72.63 & 72.44 \\
\hline benzonitrile & 86.28 & & 85.66 & 89.60 & & 88.94 & 92.13 & & 91.45 \\
\hline furan & 48.69 & 48.34 & 48.36 & 50.14 & 49.75 & 49.80 & 51.23 & 50.80 & 50.88 \\
\hline imidazole & 49.50 & & 49.17 & 51.00 & & 50.65 & 52.11 & & 51.76 \\
\hline oxazole & 43.47 & 43.18 & 43.19 & 44.63 & 44.32 & 44.35 & 45.48 & 45.16 & 45.20 \\
\hline phenol & 74.69 & & 74.15 & 77.38 & & 76.82 & 79.43 & & 78.85 \\
\hline pyrazine & 59.09 & 58.83 & 58.68 & 61.16 & 60.89 & 60.73 & 62.78 & 62.50 & 62.34 \\
\hline pyridazine & 58.97 & 58.73 & 58.56 & 60.87 & 60.62 & 60.45 & 62.33 & 62.07 & 61.89 \\
\hline pyridine & 63.64 & & 63.19 & 65.77 & & 65.31 & 67.38 & & 66.91 \\
\hline pyrimidine & 58.23 & 57.81 & 57.83 & 60.05 & 59.62 & 59.64 & 61.42 & 60.98 & 61.00 \\
\hline pyrrole & 55.01 & 54.47 & 54.64 & 56.87 & 56.25 & 56.48 & 58.27 & 57.59 & 57.86 \\
\hline phosphole & 74.18 & 73.52 & 73.65 & 77.60 & 76.85 & 77.04 & 80.32 & 79.51 & 79.74 \\
\hline thiazole & 59.35 & 58.87 & 58.93 & 61.22 & 60.69 & 60.79 & 62.61 & 62.05 & 62.17 \\
\hline thiophene & 64.37 & 63.85 & 63.91 & 66.55 & 65.98 & 66.08 & 68.20 & 67.58 & 67.72 \\
\hline
\end{tabular}

Table 4. SOPPA Static and Dynamic Isotropic Polarizabilities (in au) Obtained with the Basis Sets aug-cc-pVTZ and Sadlej

\begin{tabular}{|c|c|c|c|c|c|c|}
\hline \multirow[b]{3}{*}{ molecules } & \multicolumn{6}{|c|}{ frequency $(\mathrm{au})$} \\
\hline & \multicolumn{2}{|c|}{0.000000} & \multicolumn{2}{|c|}{0.072003} & \multicolumn{2}{|c|}{0.093215} \\
\hline & Sadlej & aug-cc-pVTZ & Sadlej & aug-cc-pVTZ & Sadlej & aug-cc-pVTZ \\
\hline benzene & 71.52 & 70.96 & 74.42 & 73.79 & 76.63 & 75.96 \\
\hline benzonitrile & 89.47 & 89.00 & 93.60 & 93.13 & 96.86 & 96.40 \\
\hline furan & 50.69 & 50.05 & 52.40 & 51.68 & 53.69 & 52.92 \\
\hline imidazole & 51.29 & 50.61 & 53.04 & 52.28 & 54.36 & 53.54 \\
\hline oxazole & 45.28 & 44.78 & 46.65 & 46.11 & 47.68 & 47.10 \\
\hline phenol & 78.33 & 77.59 & 81.77 & 80.95 & 84.48 & 83.61 \\
\hline pyrazine & 61.31 & 60.92 & 63.87 & 63.50 & 65.99 & 65.65 \\
\hline pyridazine & 61.17 & 60.79 & 63.14 & 63.53 & 65.14 & 65.50 \\
\hline pyridine & 66.29 & 65.79 & 68.90 & 68.37 & 70.91 & 70.36 \\
\hline pyrimidine & 61.09 & 60.64 & 63.38 & 62.91 & 65.17 & 64.68 \\
\hline pyrrole & 56.92 & 56.06 & 59.06 & 58.09 & 60.70 & 59.64 \\
\hline phosphole & 77.05 & 76.06 & 81.13 & 80.03 & 84.51 & 83.32 \\
\hline thiazole & 61.82 & 61.24 & 64.10 & 63.47 & 65.85 & 65.18 \\
\hline thiophene & 66.75 & 66.07 & 69.37 & 68.61 & 71.38 & 70.57 \\
\hline
\end{tabular}

3.2. CC3 Reference Values. For the benchmarking of any method, the reference values should be of a higher level of theory. Accordingly, in this study, the reference values are calculated with the coupled-cluster method CC3, which is an approximation of the full CCSDT model, where the equations of the triple amplitudes are approximated at the lowest level of perturbation theory. A huge disadvantage with this method is its long computational time, which has prevented us from obtaining CC3 results for some of the molecules in our benchmark set using the aug-cc-pVTZ basis set. For these molecules, benzonitrile, imidazole, phenol, and pyridine, the reference values must thus be obtained from a smaller basis set. From the statistical analysis of the basis sets, aug-cc-pVDZ was found to deviate less from the aug-cc-pVTZ values than Sadlej's basis set. However, because the missing reference values will be obtained as a function of the polarizability with a smaller basis set, the slope of the linear correlation is considered to be of higher importance. For this reason, Sadlej's basis set is chosen over aug-cc-pVDZ despite the statistical analysis. We will thus obtain the aug-cc-pVTZ value as a function of Sadlej's value. The correlation between the CC3 results obtained with Sadlej's and the aug-cc-pVTZ basis sets, for both the static and dynamic polarizabilities, was found to be of the linear expression

$$
y=0.9919 x+0.0693,\left(R^{2}\right)=0.9997
$$

The polarizabilities obtained with both basis sets correlate nicely, with an $R^{2}$ value close to 1 and a small offset.

Table 3 shows the CC3 polarizabilities obtained with the aug-cc-pVTZ and Sadlej's basis sets. The individual tensor components of the polarizability for this method and these two basis sets can be found in Tables S3-S8. In Table 3, in the column (aT), the "pseudo" aug-cc-pVTZ values, obtained with eq 1 from the corresponding Sadlej basis set results, are shown. These values are, as previously mentioned, used for the molecules where the polarizabilities were not obtained with the aug-cc-pVTZ basis set. As can be seen from comparing the "real" aug-cc-pVTZ results in column aT with the "pseudo" triple- $\zeta$ results in column $(\mathrm{aT})$, this approach gives almost the same polarizabilities with only slight deviations. For the static 
Table 5. SOPPA(CC2) Static and Dynamic Isotropic Polarizabilities (in au) Obtained with the Basis Sets aug-cc-pVTZ and Sadlej

\begin{tabular}{|c|c|c|c|c|c|c|}
\hline \multirow[b]{3}{*}{ molecules } & \multicolumn{6}{|c|}{ frequency $(\mathrm{au})$} \\
\hline & \multicolumn{2}{|c|}{0.000000} & \multicolumn{2}{|c|}{0.072003} & \multicolumn{2}{|c|}{0.093215} \\
\hline & Sadlej & aug-cc-pVTZ & Sadlej & aug-cc-pVTZ & Sadlej & aug-cc-pVTZ \\
\hline benzene & 71.27 & 70.91 & 74.13 & 73.71 & 76.31 & 75.85 \\
\hline benzonitrile & 89.04 & 88.75 & 93.09 & 92.78 & 96.26 & 95.95 \\
\hline furan & 50.52 & 50.05 & 52.20 & 51.66 & 53.47 & 52.88 \\
\hline imidazole & 51.18 & 50.66 & 52.91 & 52.32 & 54.22 & 53.57 \\
\hline oxazole & 45.13 & 44.78 & 46.49 & 46.09 & 47.50 & 47.07 \\
\hline phenol & 77.96 & 77.44 & 81.34 & 80.74 & 84.00 & 83.34 \\
\hline pyrazine & 61.16 & 60.91 & 63.68 & 63.43 & 65.75 & 65.52 \\
\hline pyridazine & 60.99 & 60.75 & 63.06 & 63.32 & 64.98 & 65.23 \\
\hline pyridine & 66.05 & 65.73 & 68.62 & 68.26 & 70.60 & 70.21 \\
\hline pyrimidine & 60.76 & 60.48 & 63.01 & 62.70 & 64.75 & 64.42 \\
\hline pyrrole & 56.70 & 56.05 & 58.82 & 58.06 & 60.43 & 59.58 \\
\hline phosphole & 76.70 & 75.98 & 80.71 & 79.88 & 84.00 & 83.09 \\
\hline thiazole & 61.80 & 61.44 & 64.07 & 63.66 & 65.81 & 65.35 \\
\hline thiophene & 66.52 & 66.07 & 69.10 & 68.58 & 71.09 & 70.50 \\
\hline
\end{tabular}

Table 6. SOPPA(CCSD) Static and Dynamic Isotropic Polarizabilities (in au) Obtained with the Basis Sets aug-cc-pVTZ and Sadlej

\begin{tabular}{|c|c|c|c|c|c|c|}
\hline \multirow[b]{3}{*}{ molecules } & \multicolumn{6}{|c|}{ frequency $(\mathrm{au})$} \\
\hline & \multicolumn{2}{|c|}{0.000000} & \multicolumn{2}{|c|}{0.072003} & \multicolumn{2}{|c|}{0.093215} \\
\hline & Sadlej & aug-cc-pVTZ & Sadlej & aug-cc-pVTZ & Sadlej & aug-cc-pVTZ \\
\hline benzene & 69.67 & 69.69 & 72.57 & 72.62 & 74.80 & 74.88 \\
\hline benzonitrile & 87.23 & 87.45 & 91.45 & 91.80 & 94.82 & 95.31 \\
\hline furan & 49.28 & 49.04 & 50.94 & 50.68 & 52.20 & 51.92 \\
\hline imidazole & 50.06 & 49.74 & 51.78 & 51.44 & 53.09 & 52.72 \\
\hline oxazole & 44.04 & 43.86 & 45.38 & 45.19 & 46.39 & 46.19 \\
\hline phenol & 76.03 & 75.90 & 79.48 & 79.38 & 82.24 & 82.18 \\
\hline pyrazine & 59.84 & 59.91 & 62.52 & 62.70 & 64.80 & 65.16 \\
\hline pyridazine & 59.61 & 59.68 & 62.16 & 62.02 & 64.36 & 64.08 \\
\hline pyridine & 64.59 & 64.58 & 67.22 & 67.26 & 69.28 & 69.37 \\
\hline pyrimidine & 59.47 & 59.43 & 61.78 & 61.77 & 63.59 & 63.63 \\
\hline pyrrole & 55.59 & 55.21 & 57.71 & 57.27 & 59.34 & 58.85 \\
\hline phosphole & 75.17 & 74.81 & 79.25 & 78.91 & 82.65 & 82.36 \\
\hline thiazole & 60.19 & 59.95 & 62.43 & 62.19 & 64.16 & 63.92 \\
\hline thiophene & 65.07 & 64.82 & 67.64 & 67.38 & 69.64 & 69.36 \\
\hline
\end{tabular}

Table 7. CC2 Static and Dynamic Isotropic Polarizabilities (in au) Obtained with the Basis Sets aug-cc-pVTZ and Sadlej

\begin{tabular}{|c|c|c|c|c|c|c|}
\hline \multirow[b]{3}{*}{ molecules } & \multicolumn{6}{|c|}{ frequency $(\mathrm{au})$} \\
\hline & \multicolumn{2}{|c|}{0.000000} & \multicolumn{2}{|c|}{0.072003} & \multicolumn{2}{|c|}{0.093215} \\
\hline & Sadlej & aug-cc-pVTZ & Sadlej & aug-cc-pVTZ & Sadlej & aug-cc-pVTZ \\
\hline benzene & 72.29 & 71.85 & 74.91 & 74.41 & 76.88 & 76.33 \\
\hline benzonitrile & 90.05 & 89.65 & 93.60 & 93.17 & 96.31 & 95.85 \\
\hline furan & 51.10 & 50.60 & 52.66 & 52.09 & 53.81 & 53.20 \\
\hline imidazole & 51.97 & 51.39 & 53.60 & 52.94 & 54.81 & 54.10 \\
\hline oxazole & 45.69 & 45.29 & 46.95 & 46.51 & 47.87 & 47.40 \\
\hline phenol & 79.23 & 78.63 & 82.24 & 81.58 & 84.55 & 83.83 \\
\hline pyrazine & 62.23 & 61.87 & 64.47 & 64.08 & 66.21 & 65.81 \\
\hline pyridazine & 62.06 & 61.73 & 64.14 & 63.77 & 65.73 & 65.35 \\
\hline pyridine & 67.03 & 66.61 & 69.37 & 68.91 & 71.13 & 70.64 \\
\hline pyrimidine & 61.51 & 61.14 & 63.52 & 63.12 & 65.04 & 64.61 \\
\hline pyrrole & 57.48 & 56.76 & 59.44 & 58.63 & 60.92 & 60.03 \\
\hline phosphole & 77.67 & 76.87 & 81.23 & 80.32 & 84.05 & 83.05 \\
\hline thiazole & 62.57 & 62.11 & 64.62 & 64.12 & 66.16 & 65.61 \\
\hline thiophene & 67.33 & 66.80 & 69.67 & 69.06 & 71.42 & 70.75 \\
\hline
\end{tabular}


Table 8. CCSD Static and Dynamic Isotropic Polarizabilities (in au) Obtained with the Basis Sets aug-cc-pVTZ and Sadlej

\begin{tabular}{|c|c|c|c|c|c|c|}
\hline \multirow[b]{3}{*}{ molecules } & \multicolumn{6}{|c|}{ frequency (au) } \\
\hline & \multicolumn{2}{|c|}{0.000000} & \multicolumn{2}{|c|}{0.072003} & \multicolumn{2}{|c|}{0.093215} \\
\hline & Sadlej & aug-cc-pVTZ & Sadlej & aug-cc-pVTZ & Sadlej & aug-cc-pVTZ \\
\hline benzene & 69.40 & 69.15 & 71.75 & 71.46 & 73.50 & 73.17 \\
\hline benzonitrile & 86.49 & 86.26 & 89.65 & 89.40 & 92.05 & 91.78 \\
\hline furan & 48.90 & 48.52 & 50.30 & 49.88 & 51.35 & 50.88 \\
\hline imidazole & 49.70 & 49.19 & 51.15 & 50.58 & 52.22 & 51.61 \\
\hline oxazole & 43.65 & 43.31 & 44.77 & 44.40 & 45.59 & 45.20 \\
\hline phenol & 75.33 & 74.90 & 77.95 & 77.46 & 79.93 & 79.40 \\
\hline pyrazine & 59.53 & 59.28 & 61.54 & 61.27 & 63.09 & 62.81 \\
\hline pyridazine & 59.36 & 59.12 & 61.19 & 60.93 & 62.58 & 62.29 \\
\hline pyridine & 64.20 & 63.91 & 66.28 & 65.97 & 67.84 & 67.50 \\
\hline pyrimidine & 58.80 & 58.50 & 60.59 & 60.25 & 61.92 & 61.56 \\
\hline pyrrole & 55.30 & 54.72 & 57.11 & 56.45 & 58.46 & 57.74 \\
\hline phosphole & 74.90 & 74.27 & 78.26 & 77.52 & 80.92 & 80.10 \\
\hline thiazole & 59.71 & 59.23 & 61.52 & 60.98 & 62.87 & 62.28 \\
\hline thiophene & 64.82 & 64.34 & 66.95 & 66.39 & 68.54 & 67.93 \\
\hline
\end{tabular}

case, the absolute mean deviation is $0.11 \mathrm{au}$, and the largest deviation is found for benzene, with a deviation of $0.31 \%$ from the aug-cc-pVTZ value. For the dynamic case, the absolute mean deviation is $0.14 \mathrm{au}$, with pyrrole at 0.093215 au having the largest deviation of $0.47 \%$.

To verify that the slope is of more importance when predicting values from a linear fit, the same approach was applied to the aug-cc-pVDZ basis set results. The CC3 polarizabilities obtained with the aug-cc-pVDZ basis set can be found in Table S9. It was found that the aug-cc-pVTZ values predicted from the aug-cc-pVDZ values deviated more from the true aug-cc-pVTZ values. For the static case, the absolute mean deviation was $0.45 \mathrm{au}$, with pyrimidine having the largest deviation of $0.95 \%$ from the true value. For the dynamic case, the absolute mean deviation was 0.43 au, with the most deviating result belonging to pyrimidine at 0.072003 au with a $0.92 \%$ deviation.

It can thus safely be concluded that for such predictions, the slope is of higher importance for the accuracy. Because the deviations for Sadlej's polarized triple- $\zeta$ basis set are relatively small, the basis set is found to be a valuable alternative in combination with the CC3 method instead of using a larger basis set with a method of a lower level of theory.

3.3. Benchmarking of Methods. Having found appropriate reference values (Table 3 ), the performance of different second-order methods is now investigated using the aug-ccpVTZ basis set. Values of the polarizabilities obtained from the different methods using the aug-cc-pVTZ and Sadlej basis sets can be found in Tables 4-8.

In Figure 2, the mean and absolute mean deviations of the results of the different methods from the CC3 results are shown for the static case. The numerical values for the deviations can be seen in Table 9. The method giving the results that deviate most from the $\mathrm{CC} 3$ results is found to be $\mathrm{CC} 2$, with a mean deviation of $3.06 \pm 0.68$ au. One might have expected CC2 to perform worse than CCSD, with a mean deviation of $0.45 \pm 0.24 \mathrm{au}$, because the equations for the double amplitudes are in CC2 approximated based on MøllerPlesset theory. However, this is in contrast with the previous results for singlet excitation energies, ${ }^{8-10}$ where CC2, on average, performed better than CCSD in comparison with CC3. SOPPA is found to yield results that deviate less from the CC3 reference values than CC2, with a mean deviation of 2.30

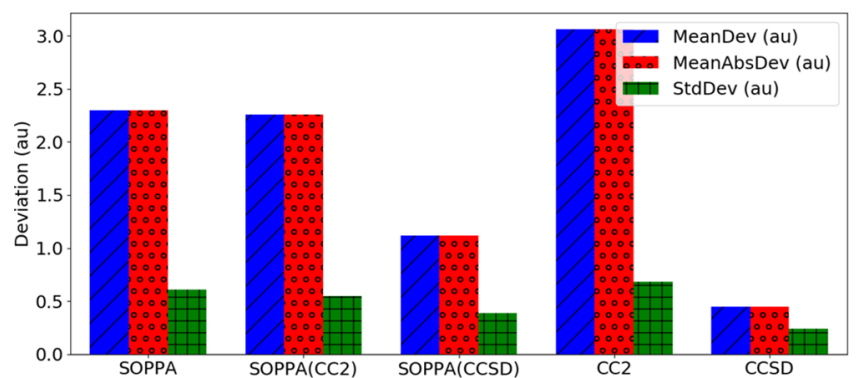

Figure 2. Deviation from CC3 results for static polarizabilities (in au).

$\pm 0.61 \mathrm{au}$, which suggests using SOPPA instead of CC2 for calculations of polarizabilities. A similar better performance of SOPPA than CC2 in comparison with CCSD values was also previously found for NMR spin-spin coupling constants. ${ }^{12}$ For vertical excitation energies, on the contrary, CC2 has been shown to yield results in clearly better agreement with CC3 results than SOPPA. ${ }^{24,68,69}$ It is then seen for the mixed methods, SOPPA(CC2) and SOPPA(CCSD), that the SOPPA results are improved by using the coupled-cluster amplitudes in the wave functions instead of the Møller-Plesset correlation coefficients, making SOPPA(CC2), with a mean deviation of $2.26 \pm 0.55 \mathrm{au}$, slightly better than SOPPA and making SOPPA (CCSD) the best, with a mean deviation of $1.12 \pm 0.39$ $\mathrm{au}$. The fact that the mean and absolute mean deviation are the same for the SOPPA, SOPPA(CC2), SOPPA(CCSD), and CC2 methods shows that all of these methods consistently overestimate the CC3 static polarizabilities.

The same overall performance of the methods is also observed for the frequency-dependent polarizabilities, which can be seen in Figure 3 and in Table 9. However, the differences in performances between $\mathrm{CC} 2$ and the SOPPA methods are less pronounced here; CC2's mean deviation is $3.27 \pm 0.76$ au versus $2.97 \pm 0.89$ au for SOPPA, $2.86 \pm 0.79$ au for SOPPA(CC2), and $1.89 \pm 0.79$ au for SOPPA(CCSD). Furthermore, the mean deviations are actually larger than those for the static polarizabilities. CCSD still leads to the results closest to the CC3 results, with an actually smaller mean deviation of $0.34 \pm 0.23$ au than for the static polarizabilities. The mean and absolute mean deviations here again show that these methods mostly overestimate the CC3 results. The 
Table 9. Deviations in Static and Dynamic Polarizabilities (in au) of All Methods with Respect to the CC3/aT or CC3/(aT) Results $^{a}$

\begin{tabular}{|c|c|c|c|c|c|c|}
\hline \multirow[b]{2}{*}{ method } & \multicolumn{3}{|c|}{ static } & \multicolumn{3}{|c|}{ dynamic } \\
\hline & mean & abs. mean & std. dev. & mean & abs. mean & std. dev. \\
\hline SOPPA & 2.30 & 2.30 & 0.61 & 2.97 & 2.97 & 0.90 \\
\hline SOPPA(CC2) & 2.26 & 2.26 & 0.55 & 2.86 & 2.86 & 0.79 \\
\hline SOPPA(CCSD) & 1.12 & 1.12 & 0.39 & 1.89 & 1.89 & 0.79 \\
\hline $\mathrm{CC} 2$ & 3.06 & 3.06 & 0.68 & 3.27 & 3.27 & 0.76 \\
\hline CCSD & 0.45 & 0.45 & 0.24 & 0.34 & 0.35 & 0.23 \\
\hline
\end{tabular}

${ }^{a}$ Dynamic polarizabilities for the frequencies 0.072003 and 0.93215 au.

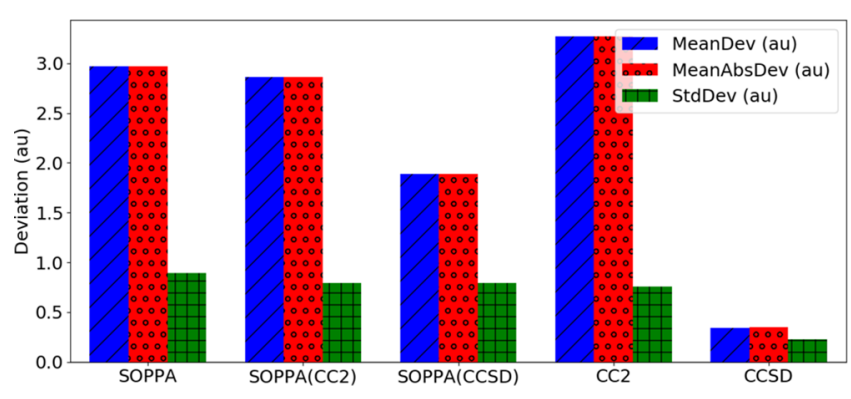

Figure 3. Deviation from CC3 results for frequency-dependent polarizabilities (in au). The dynamic polarizabilities are of frequencies 0.072003 and 0.93215 au.

exception is the frequency-dependent polarizabilities of imidazole calculated with the CCSD method. Unlike all other cases, here the CCSD method underestimates the polarizability compared with the value obtained from CC3, which can be seen in Table 8 .

Because few of the reference CC3 polarizabilities are not true aug-cc-pVTZ values but are obtained through eq 1 from results using the Sadlej basis set, the previous discussion is not a completely true analysis of the methods. For that reason, the same analyses is made here again for all methods using only the true CC3/aug-cc-pVTZ values. Although this will represent the methods better because they are compared on exactly the same level (aug-cc-pVTZ), it is worth remembering that the test set is now smaller. Figures 4 and 5 show the mean

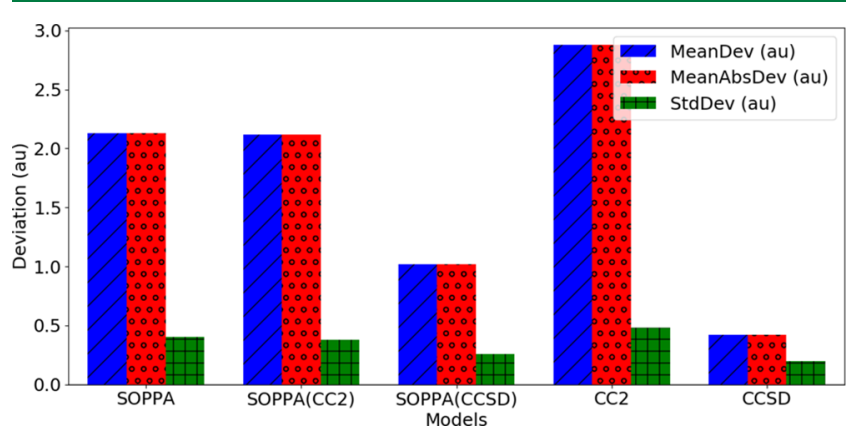

Figure 4. Deviations from only true CC3/aug-cc-pvTZ results for static polarizabilities (in au).

deviations for the different methods from only the CC3 polarizabilities, which were obtained with the aug-cc-pVTZ basis set. The numerical values from the figures can be found in Table 10. At a first glance of the figures, the hierarchy of the methods is seen to be unchanged for both the static and dynamic polarizabilities. It is observed that the mean deviations, excluding the pseudo values, are lower than when

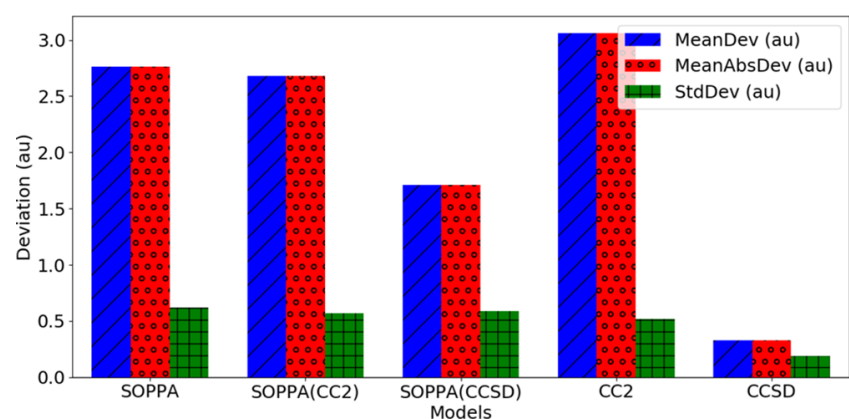

Figure 5. Deviations from only true CC3/aug-cc-pvTZ results for dynamic polarizabilities (in au). The dynamic polarizabilities are of frequencies 0.072003 and 0.93215 au.

the pseudo values are included in the analyses. It can also be seen that the difference in the methods is slightly smaller, more so for the dynamic polarizabilities. The fact that the hierarchy is the same whether or not the pseudo CC3/aug-cc-pVTZ values are included in the analyses shows that the pseudo polarizabilities can be used to rank the performance of the different methods. Furthermore, the fact that the deviations are slightly higher for the comparison including the pseudo values could simply be due to the larger data set.

From the analyses, both excluding and including the pseudo CC3/aug-cc-pVTZ polarizabilities, it can be concluded that for both static and dynamic polarizabilities it is justified to prefer SOPPA over CC2 because it yields results closer to the CC3 results. Both SOPPA and CC2 have an $N^{5}$ scaling, which makes the methods formally equally demanding. However, there are differences in the size and the number of sets of the inhomogeneous linear equations, which have to be solved in the two methods. Furthermore, in general, optimized implementations of the CC2 method in different quantumchemical computer programs have attracted more attention. Exploiting, for example, the resolution of the identity approximation, ${ }^{34}$ leading to RI-CC $2,{ }^{35}$ significantly reduces the spent CPU time. A comparison of timings between such an optimized CC2 implementation and our standard SOPPA implementation is therefore not meaningful. Had the implementation of the SOPPA method received as much attention, SOPPA calculations with a tuned SOPPA program could potentially run as fast as CC2 calculations with current RI-CC2 programs. For even closer agreement with the CCSD or CC3 results, the ground-state amplitudes of the coupledcluster methods can be used in the SOPPA approach, leading to the SOPPA(CC2) or SOPPA(CCSD) methods. The order of increasing performance is thus CC2 < SOPPA < SOPPA $(\mathrm{CC} 2)<\operatorname{SOPPA}(\mathrm{CCSD})<\mathrm{CCSD}$. It is worth mentioning that these mixed methods, SOPPA(CC2) and 
Table 10. Deviations in Static and Dynamic Polarizabilities (in au) of All Methods with Respect to the True CC3/aT Results Only $^{a}$

\begin{tabular}{|c|c|c|c|c|c|c|}
\hline \multirow[b]{2}{*}{ method } & \multicolumn{3}{|c|}{ static } & \multicolumn{3}{|c|}{ dynamic } \\
\hline & mean & abs. mean & std. dev. & mean & abs. mean & std. dev. \\
\hline SOPPA & 2.13 & 2.13 & 0.40 & 2.76 & 2.76 & 0.62 \\
\hline SOPPA(CC2) & 2.12 & 2.12 & 0.38 & 2.68 & 2.68 & 0.57 \\
\hline SOPPA(CCSD) & 1.02 & 1.02 & 0.26 & 1.71 & 1.71 & 0.59 \\
\hline $\mathrm{CC} 2$ & 2.88 & 2.88 & 0.48 & 3.06 & 3.06 & 0.52 \\
\hline CCSD & 0.42 & 0.42 & 0.20 & 0.33 & 0.33 & 0.19 \\
\hline
\end{tabular}

${ }^{a}$ Dynamic polarizabilities being of frequencies: 0.072003 and 0.93215 au.

Table 11. Static Experimental Polarizabilites (in au): Absolute Deviations from Experimental Values (in au) for the Different Methods $^{a}$

\begin{tabular}{|c|c|c|c|c|c|c|c|}
\hline molecule & exp. value & SOPPA & SOPPA(CC2) & SOPPA(CCSD) & $\mathrm{CC} 2$ & CCSD & $\mathrm{CC} 3$ \\
\hline benzene & $67.48^{b}$ & 3.48 & 3.43 & 2.21 & 4.37 & 1.67 & 1.01 \\
\hline pyrrole & $53.47^{c}$ & 2.59 & 2.58 & 1.74 & 3.29 & 1.25 & 1.00 \\
\hline thiophene & $65.18^{d}$ & 0.89 & 0.89 & 0.36 & 1.62 & 0.84 & 1.33 \\
\hline furan & $48.59^{e}$ & 1.46 & 1.46 & 0.45 & 2.01 & 0.07 & 0.25 \\
\hline pyridine & $64.11^{f}$ & 1.68 & 1.62 & 0.47 & 2.50 & 0.20 & 0.94 \\
\hline pyrimidine & $59.35^{f}$ & 1.29 & 1.13 & 0.08 & 1.79 & 0.85 & 1.54 \\
\hline pyrazine & $60.62^{f}$ & 0.30 & 0.29 & 0.71 & 1.25 & 1.34 & 1.79 \\
\hline pyridazine & $59.32^{f}$ & 1.47 & 1.43 & 0.36 & 2.41 & 0.20 & 0.59 \\
\hline mean abs. dev. & & 1.65 & 1.60 & 0.80 & 2.40 & 0.80 & 1.05 \\
\hline
\end{tabular}

Table 12. Dynamic Polarizabilities (in au): Absolute Deviations from Experimental Values ${ }^{a}$

\begin{tabular}{|c|c|c|c|c|c|c|c|}
\hline \multicolumn{8}{|c|}{ frequency: $0.072003 \mathrm{au}$} \\
\hline molecule & exp. value & SOPPA & SOPPA(CC2) & SOPPA(CCSD) & $\mathrm{CC} 2$ & CCSD & $\mathrm{CC} 3$ \\
\hline \multirow[t]{2}{*}{ furan } & $49.1 \pm 2.2^{b}$ & 2.58 & 2.56 & 1.58 & 2.99 & 0.78 & 0.65 \\
\hline & $49.1 \pm 0.5^{b}$ & 2.58 & 2.56 & 1.58 & 2.99 & 0.78 & 0.65 \\
\hline \multirow[t]{2}{*}{ thiophene } & $65.2 \pm 2.1^{c}$ & 3.41 & 3.38 & 2.18 & 3.86 & 1.19 & 0.78 \\
\hline & $64.9 \pm 0.6^{c}$ & 3.71 & 3.68 & 2.48 & 4.16 & 1.49 & 1.08 \\
\hline mean abs. dev. & & 3.07 & 3.04 & 1.95 & 3.50 & 1.06 & 0.79 \\
\hline
\end{tabular}

SOPPA(CCSD), require more CPU time than SOPPA in the calculation of the wave-function amplitudes but are still faster than their corresponding coupled-cluster methods, CC2 and CCSD, assuming a comparable implementation. Whereas this is true for the molecules studied here, the difference in timing between SOPPA(CCSD) and CCSD is not guaranteed to remain the same for larger molecules. The calculation of the response functions of SOPPA(CCSD) scales as SOPPA, that is, $N^{5}$, but the calculation of the wave-function amplitudes scales as $N^{6}$. For larger molecules, the amplitude scaling could start to dominate, thus decreasing the difference in CPU time between SOPPA(CCSD) and CCSD. ${ }^{70}$

3.4. Comparison with Experiment. Experimental values for static polarizabilities were found for benzene, pyrrole, thiophene, furan, pyridine, pyrimidine, pyrazine, and pyridazine. For furan and thiophene, we also found two experimental values for the frequency 0.072003 au. The calculated polarizabilities, with the aug-cc-pVTZ basis set, are then compared with the experimental values to obtain an alternative criteria for the accuracy of the different theoretical methods. In Table 11, the absolute deviations of the different methods can be seen for the static polarizabilities. From the absolute mean deviations, the second-order methods are found to show the same trend as when compared with the calculated $\mathrm{CC} 3$ values. The CCSD method along with SOPPA(CCSD) yields results, on average, closest to the experimental values with an absolute mean deviation of 0.80 au. SOPPA (absolute mean deviation $1.65 \mathrm{au})$ performs better than CC2 $(2.40 \mathrm{au})$, and the performance of the SOPPA method can be further improved by using the mixed methods: SOPPA(CC2) (1.60 au) of SOPPA(CCSD) $(0.80 \mathrm{au})$. SOPPA(CCSD) is, in this comparison, thus performing as good as CCSD, on average. This tendency is similar to the one observed in the study by Hodecker et al., ${ }^{26}$ which showed the same tendency of improving the results when the CC amplitudes were included in $\mathrm{ADC}$ second-order methods. They also found that the implementation of the CC amplitudes for similar systems lowered the polarizability, thus reducing the deviations from experiment. The CC3 results, compared with experimental values, are, however, not the closest ones, with an absolute mean deviation of $1.05 \mathrm{au}$. The performance is very close to CCSD and SOPPA(CCSD) but does have the largest absolute mean deviation of the three methods. This unexpected performance of CC3 in comparison with the experimental values could be a consequence of missing vibrational corrections in the calculation, which have a greater influence on the static case, as there exists a purely vibrational contribution. These purely vibrational contribution can, however, be neglected in the dynamic case. A second reason for the deviations might be the geometries of the molecules, 
which, in this study, were optimized at only the MP2/6$31 \mathrm{G}(\mathrm{d})$ level.

In Table 12, the absolute deviations for furan and thiophene are shown for the dynamic polarizabilities. Here the same order of performance is seen as for the static case, apart from the fact that the $\mathrm{CC} 3$ method is now reproducing the experimental values the best. It is worth noticing that the experimental values for furan and thiophene in cyclohexane solutions have larger uncertainties, whereas for the pure solvents, the uncertainties are smaller. This illustrates the effect of the solvent, which is neglected in the calculations.

The test set of molecules here is much too small to be truly representative, and thus the tendency could change if more molecules were included. Nevertheless, although the test set is small for testing the performance of the methods against experimental values, it does point to a conclusion. It seems that the trend in the performance of the second-order methods is the same independent of whether the reference data are calculated CC3 values or experimental data. However, to surely prove this, the set of experimental values needs to be expanded. Another question that should be considered is whether the experimental values are even really representative. For the experimental polarizabilities, multiple factors can have an influence on the value as uncertainty on the apparatus used, purity of the product, solvent effects, or vibrational averaging corrections. All of these factors are not accounted for in our calculated polarizabilities. Furthermore, the ground-state structure of the molecule could have a big influence on the calculated polarizability, which, in our case, was optimized at only the MP2/6-31G(d) level. For these reasons, a comparison of calculated and experimentally obtained polarizabilities is not truly based on the same assumptions.

\section{CONCLUSIONS}

A benchmark of frequency-dependent polarizabilities using the second-order methods SOPPA, SOPPA(CC2), SOPPA(CCSD), CC2, and CCSD was made. For the test set of 14 (hetero)aromatic medium-sized organic molecules, CC3 values for the static and frequency-dependent polarizabilities were obtained for the first time using Sadlej's polarized triple- $\zeta$ basis set and, for a subset of the molecules, also using the aug-ccpVTZ basis set. These CC 3 values were then used as reference values for the benchmark, showing that CCSD performed best in comparison with CC3. This is in contradiction with previous findings for vertical excitation energies, where CC2 was found to yield values closer to the CC 3 values than CCSD. The SOPPA method was found to perform better than CC2, and its performance could be further enhanced by employing coupledcluster amplitudes in the wave function.

Furthermore, for this set of molecules, the effect of different basis sets was tested at the SOPPA level. The basis sets in focus were Dunning's aug-cc-pVDZ, aug-cc-pVTZ, and aug-ccpVQZ and the double-augmented d-aug-cc-pVTZ. Moreover Sadlej's polarized triple- $\zeta$ basis set, which is specialized for polarizabilities, was also tested. It was found that for static polarizabilities, all basis sets performed similarly. For the frequency-dependent polarizabilities, a greater influence of the basis sets was seen. The aug-cc-pVTZ was found to perform closely to the aug-cc-pVQZ, with only a slight offset, and the difference between aug-cc-pVTZ and d-aug-cc-pVTZ was found to be insignificant, and thus no real gain is achieved using these basis sets. Sadlej's polarized triple- $\zeta$ basis set was found to deviate more than the aug-cc-pVDZ from the aug-cc-
pVTZ basis set, but linear correlations showed that Sadlej's basis set had a slope closer to 1 with the aug-cc-pVTZ basis set than with the aug-cc-pVDZ basis set. Thus for a wellperforming basis set for both static and frequency-dependent polarizabilities, the aug-cc-pVTZ was found to be adequate.

For a small set of the molecules, a comparison of calculated polarizabilities with experimental values was made. Although the set was small, the tendency for the second-order methods does point toward the same tendency, as is seen when the reference values are CC3 polarizabilities.

\section{ASSOCIATED CONTENT}

\section{Supporting Information}

The Supporting Information is available free of charge at https://pubs.acs.org/doi/10.1021/acs.jctc.9b01300.

Tables S1 and S2. Static and dynamic polarizabilities calculated with the SOPPA method using the basis sets aug-cc-pVDZ, aug-cc-pVTZ, d-aug-cc-pVTZ, aug-ccpVQZ, and sadlej-pVTZ. Tables S3-S8. Individual tensor components of the polarizability for the CC3 method using the aug-cc-pVTZ and Sadlej's basis sets. Table S9. CC3 static and dynamic polarizabilities obtained with the aug-cc-pVDZ basis and the "pseudo" aug-cc-pVTZ values predicted from the aug-cc-pVDZ basis set. Figure S1. Linear correlations described in Table 1 for static polarizabilities. Figure S2. Correlations from the same table for the dynamic polarizabilities (PDF)

\section{AUTHOR INFORMATION}

\section{Corresponding Author}

Stephan P. A. Sauer - Department of Chemistry, University of Copenhagen, Copenhagen 2100, Denmark; $\odot$ orcid.org/00000003-4812-0522; Email: sauer@kiku.dk

\section{Authors}

Maria W. Jørgensen - Department of Chemistry, University of Copenhagen, Copenhagen 2100, Denmark

Rasmus Faber - Department of Chemistry, Technical University of Denmark, Kongens Lyngby 2800, Denmark; 이이.org/ 0000-0001-8524-0684

Andrea Ligabue - Game Science Research Center, University of Modena and Reggio Emilia, Modena 41121, Italy

Complete contact information is available at:

https://pubs.acs.org/10.1021/acs.jctc.9b01300

\section{Notes}

The authors declare no competing financial interest.

\section{ACKNOWLEDGMENTS}

We thank the Department of Chemistry, University of Copenhagen for the access to its high-performance computer cluster. Christoffer H. S. Møller is gratefully acknowledged for providing the Python code for all graphs in this work and for adapting the code to the wishes of the authors.

\section{REFERENCES}

(1) Böttcher, C. J. F.; van Belle, O. C.; Bordewijk, P.; Rip, A. Theory of Electric Polarization; Elsevier Science, Ltd., 1978; Vol. 2.

(2) Litchinitser, N. M. Nonlinear optics in metamaterials. $A D V$. PHYS: X 2018, 3, 701-719. 
(3) Oddershede, J.; Svendsen, E. N. Dynamic polarizabilities and Raman intensities of $\mathrm{CO}, \mathrm{N}_{2}, \mathrm{HCl}$ and $\mathrm{Cl}_{2}$. Chem. Phys. 1982, 64, 359-369.

(4) Paidarová, I.; Curík, R.; Sauer, S. P. A. Calculations of polarizabilities and their gradients for electron energy-loss spectroscopy. Collect. Czech. Chem. Commun. 2008, 73, 1509-1524.

(5) Paidarová, I.; Sauer, S. P. A. A comparison of density functional theory and coupled cluster methods for the calculation of electric dipole polarizability gradients of methane. AIP Conf. Proc. 2009, 1504, 695-698.

(6) Sauer, S. P. A.; Paidarová, I.; Čársky, P.; Čurík, R. Calculation of dipole polarizability derivatives of adamantane and their use in electron scattering computations. Eur. Phys. J. D 2016, 70, 105.

(7) Malvaldi, M.; Persico, M.; Van Leuven, P. Infrared multiphoton absorption and electronic polarizability. J. Chem. Phys. 1999, 111, 9560-9567.

(8) Schreiber, M.; Silva-Junior, M. R.; Sauer, S. P. A.; Thiel, W. Benchmarks for electronically excited states: CASPT2, CC2, CCSD, and CC3. J. Chem. Phys. 2008, 128, 134110.

(9) Sauer, S. P. A.; Schreiber, M.; Silva-Junior, M. R.; Thiel, W. Benchmarks for Electronically Excited States: A Comparison of Noniterative and Iterative Triples Corrections in Linear Response Coupled Cluster Methods: CCSDR(3) versus CC3. J. Chem. Theory Comput. 2009, 5, 555-564.

(10) Silva-Junior, M. R.; Sauer, S. P. A.; Schreiber, M.; Thiel, W. Basis set effects on coupled cluster benchmarks of electronically excited states: CC3, CCSDR(3) and CC2. Mol. Phys. 2010, 108, 453-465.

(11) Maximoff, S. N.; Peralta, J. E.; Barone, V.; Scuseria, G. E. Assessment of Density Functionals for Predicting One-Bond CarbonHydrogen NMR Spin-Spin Coupling Constants. J. Chem. Theory Comput. 2005, 1, 541-545.

(12) Kjær, H.; Sauer, S. P. A.; Kongsted, J. Benchmarking NMR indirect nuclear spin-spin coupling constants: SOPPA, SOPPA(CC2), and SOPPA(CCSD) versus CCSD. J. Chem. Phys. 2010, 133, 144106.

(13) Kupka, T.; Nieradka, M.; Stachow, M.; Pluta, T.; Nowak, P.; Kjær, H.; Kongsted, J.; Kaminsky, J. Basis Set Convergence of Indirect Spin-Spin Coupling Constants in the Kohn-Sham Limit for Several Small Molecules. J. Phys. Chem. A 2012, 116, 3728-3738.

(14) Faber, R.; Sauer, S. P. A.; Gauss, J. The Importance of Triples Contributions to NMR Spin-spin Coupling Constants Computed at the CC3 and CCSDT Levels. J. Chem. Theory Comput. 2017, 13, 696-709.

(15) Larsen, H.; Olsen, J.; Hättig, C.; Jørgensen, P.; Christiansen, O.; Gauss, J. Polarizabilities and first hyperpolarizabilities of HF, Ne, and $\mathrm{BH}$ from full configuration interaction and coupled cluster calculations. J. Chem. Phys. 1999, 111, 1917-1925.

(16) Christiansen, O.; Hättig, C.; Gauss, J. Polarizabilities of CO, $\mathrm{N}_{2}, \mathrm{HF}, \mathrm{Ne}, \mathrm{BH}$, and $\mathrm{CH}^{+}$from ab initio calculations: Systematic studies of electron correlation, basis set errors, and vibrational contributions. J. Chem. Phys. 1998, 109, 4745-4757.

(17) Kobayashi, R.; Koch, H.; Jørgensen, P.; Lee, T. J. Comparison of coupled-cluster and Brueckner coupled-cluster calculations of molecular properties. Chem. Phys. Lett. 1993, 211, 94-100.

(18) Nielsen, E. S.; Jørgensen, P.; Oddershede, J. Transition moments and dynamic polarizabilities in a second order polarization propagator approach. J. Chem. Phys. 1980, 73, 6238.

(19) Sauer, S. P. A.; Diercksen, G. H. F.; Oddershede, J. SecondOrder Polarization Propagator Calculations of Dynamic Dipole Polarizabilities and C6 Coefficients. Int. J. Quantum Chem. 1991, 39, 667-679.

(20) Christiansen, O.; Koch, H.; Jørgensen, P. The second-order approximative coupled cluster singles and doubles model CC2. Chem. Phys. Lett. 1995, 243, 409-418.

(21) Schirmer, J. Beyond the random-phase approximation: A new approximation scheme for the polarization propagator. Phys. Rev. A: At., Mol., Opt. Phys. 1982, 26, 2395.

(22) Fransson, T.; Rehn, D. R.; Dreuw, A.; Norman, P. Static polarizabilities and $\mathrm{C}_{6}$ dispersion coefficients using the algebraic- diagrammatic construction scheme for the complex polarization propagator. J. Chem. Phys. 2017, 146, No. 094301.

(23) Sauer, S. P. A. Second-order polarization propagator approximation with coupled-cluster singles and doubles amplitudes - SOPPA(CCSD): the polarizability and hyperpolarizability of $\mathrm{Li}^{-} . J$. Phys. B: At., Mol. Opt. Phys. 1997, 30, 3773.

(24) Sauer, S. P. A.; Pitzner-Frydendahl, H. F.; Buse, M.; Jensen, H. J. A.; Thiel, W. Performance of SOPPA based methods in the calculation of vertical excitation energies and oscillator strengths. Mol. Phys. 2015, 113, 2026-2045.

(25) Hodecker, M.; Dempwolff, A. L.; Rehn, D. R.; Dreuw, A. Algebraic-diagrammatic construction scheme for the polarization propagator including ground-state coupled-cluster amplitudes. I. Excitation energies. J. Chem. Phys. 2019, 150, 174104.

(26) Hodecker, M.; Rehn, D. R.; Norman, P.; Dreuw, A. Algebraicdiagrammatic construction scheme for the polarization propagator including ground-state-coupled-cluster amplitudes. II. Static polarizabilities. J. Chem. Phys. 2019, 150, 174105.

(27) Packer, M. J.; Dalskov, E. K.; Sauer, S. P. A.; Oddershede, J. Correlated dipole polarizabilities and dipole moments of the halides $\mathrm{HX}$ and $\mathrm{CH}_{3} \mathrm{X}(\mathrm{X}=\mathrm{F}, \mathrm{Cl}$ and $\mathrm{Br})$. Theor. Chem. Acc. 1994, 89, 323.

(28) Dalskov, E. K.; Sauer, S. P. A. Correlated, Static and Dynamic Polarizabilities of Small Molecules, Comparison of Four "Black Box" Methods. J. Phys. Chem. A 1998, 102, 5269.

(29) Sauer, S. P. A.; Oddershede, J. Correlated Polarization Propagator Calculations of Static Dipole Polarizabilities. Int. J. Quantum Chem. 1994, 50, 317-332.

(30) Paidarová, I.; Sauer, S. P. A. Calculations of Dipole and Quadrupole Polarizability Radial Functions for LiH and HF: A Comparison of Different Linear Response Methods. Adv. Quantum Chem. 2005, 48, 185-208.

(31) Sauer, S. P. A.; Paidarová, I. Correlated linear response calculations of the C6 dispersion coefficients of hydrogen halides. Comput. Lett. 2007, 3, 399-421.

(32) Paidarová, I.; Sauer, S. P. A. A comparison of Møller-Plesset and coupled cluster linear response theory methods for the calculation of dipole oscillator strength sum rules and C6 dispersion coefficients. Collect. Czech. Chem. Commun. 2008, 73, 1415-1436.

(33) Pedersen, T. B.; Sánchez de Merás, A. M. J.; Koch, H. Polarizability and optical rotation calculated from the approximate coupled cluster singles and doubles CC2 linear response theory using Cholesky decompositions. J. Chem. Phys. 2004, 120, 8887-8897.

(34) Vahtras, O.; Almlöf, J.; Feyereisen, M. W. Integral approximation for LCAO-SCF calculations. Chem. Phys. Lett. 1993, 213, 514-518.

(35) Hättig, C.; Weigend, F. CC2 excitation energy calculations on large molecules using the resolution of the identity approximation. J. Chem. Phys. 2000, 113, 5154-5161.

(36) Parkinson, W. A.; Sauer, S. P. A.; Oddershede, J.; Bishop, D. M. Calculation of the Verdet Constant for $\mathrm{H}_{2}, \mathrm{~N}_{2}, \mathrm{CO}$ and FH. J. Chem. Phys. 1993, 98, 487-495.

(37) Hickey, A. L.; Rowley, C. N. Benchmarking Quantum Chemical Methods for the Calculation of Molecular Dipole Moments and Polarizabilities. J. Phys. Chem. A 2014, 118, 3678-3687.

(38) Kobayashi, R.; Koch, H.; Jørgensen, P. Calculation of frequency-dependent polarizabilities using coupled-cluster response theory. Chem. Phys. Lett. 1994, 219, 30-35.

(39) Kobayashi, R.; Koch, H.; Jørgensen, P. Static polarizabilities and dipole moment derivatives for the closed shell coupled cluster singles and doubles wave function. J. Chem. Phys. 1994, 101, 49564963.

(40) Kobayashi, R.; Amos, R. D.; Koch, H.; Jørgensen, P. Dynamic CCSD polarizabilities of $\mathrm{CHF}_{3}$ and $\mathrm{CHCl}_{3}$. Chem. Phys. Lett. 1996, 253, 373-376.

(41) Rozyczko, P. B.; Perera, S. A.; Nooijen, M.; Bartlett, R. J. Correlated calculations of molecular dynamic polarizabilities. J. Chem. Phys. 1997, 107, 6736-6747. 
(42) Hättig, C.; Christiansen, O.; Jørgensen, P. Cauchy moments and dispersion coefficients using coupled cluster linear response theory. J. Chem. Phys. 1997, 107, 10592-10598.

(43) Bokhan, D.; Trubnikov, D. N.; Perera, A.; Bartlett, R. J. Explicitly correlated coupled-cluster theory for static polarizabilities. $J$. Chem. Phys. 2016, 145, 134104.

(44) Stanton, J. F.; Bartlett, R. J. A coupled-cluster based effective Hamiltonian method for dynamic electric polarizabilities. J. Chem. Phys. 1993, 99, 5178-5183.

(45) Christiansen, O.; Hättig, C.; Jørgensen, P. Ground and excited state polarizabilities and dipole transition properties of benzene from coupled cluster response theory. Spectrochim. Acta, Part A 1999, 55, 509-524.

(46) García Cuesta, I.; Bondo Pedersen, T.; Koch, H.; Sanchez de Meras, A.M.J. Polarizabilities of small annulenes from Cholesky CC2 linear response theory. Chem. Phys. Lett. 2004, 390, 170-175.

(47) Coriani, S.; Fransson, T.; Christiansen, O.; Norman, P. Asymmetric-Lanczos-Chain-Driven Implementation of Electronic Resonance Convergent Coupled-Cluster Linear Response Theory. J. Chem. Theory Comput. 2012, 8, 1616-1628.

(48) Sadlej, A. J. Medium Size Polarized Basis Sets for High Level Correlated Calculations of Molecular Electric Properties. Collect. Czech. Chem. Commun. 1988, 53, 1995-2016.

(49) Sadlej, A. J. Medium-size polarized basis sets for high-levelcorrelated calculations of molecular electric properties. Theor Chim Acta 1992, 81, 339-354.

(50) Woon, E. D.; Dunning, T. H. Gaussian basis sets for use in correlated molecular calculations. III. The atoms aluminum through argon. J. Chem. Phys. 1993, 98, 1358-1371.

(51) Dunning, T. H. Gaussian basis sets for use in correlated molecular calculations. I. The atoms boron through neon and hydrogen. J. Chem. Phys. 1989, 90, 1007-1023.

(52) Woon, E. D.; Dunning, T. H. Gaussian basis sets for use in correlated molecular calculations. IV. Calculation of static electric response properties. J. Chem. Phys. 1994, 100, 2975-2988.

(53) Christiansen, O.; Halkier, A.; Jørgensen, P. Coupled cluster calculation of the polarizability of furan. Chem. Phys. Lett. 1997, 281, 438-444.

(54) García Cuesta, I.; Sánchez Marín, J.; Bondo Pedersen, T.; Koch, H.; Sánchez de Merás, A. M. J. Variation of polarizability in the [4n + 2] annulene series: from [22]- to [66]-annulene. Phys. Chem. Chem. Phys. 2008, 10, 361-365.

(55) Skrzyński, G.; Radula-Janik, K.; Kupka, T.; Pluta, T. Dynamic Polarizability and Higher-Order Electric Properties of Fluorene, Carbazole, and Dibenzofuran. J. Phys. Chem. A 2019, 123, 97539762.

(56) Van Caillie, C.; Amos, R. D. Static and dynamic polarisabilities, Cauchy coefficients and their anisotropies: an evaluation of DFT functionals. Chem. Phys. Lett. 2000, 328, 446-452.

(57) Adamo, C.; Cossi, M.; Scalmani, G.; Barone, V. Accurate static polarizabilities by density functional theory: assessment of the PBE0 model. Chem. Phys. Lett. 1999, 307, 265-271.

(58) Alipour, M.; Mohajeri, A. Linear optical properties and their bond length dependence of uttrium bromide from $a b$ initio and density functional theory calculations. Chem. Phys. 2011, 387, 5-10.

(59) Christiansen, O.; Koch, H.; Jørgensen, P. Response functions in the CC3 iterative triple excitation model. J. Chem. Phys. 1995, 103, 7429-7441.

(60) Noga, J.; Bartlett, R. J. The full CCSDT model for molecular electronic structure. J. Chem. Phys. 1987, 86, 7041-7050.

(61) Christiansen, O.; Gauss, J.; Stanton, J. F. The effect of triple excitations in coupled cluster calculations of frequency-dependent polarizabilities. Chem. Phys. Lett. 1998, 292, 437-446.

(62) Frisch, M. J.; Trucks, G. W.; Schlegel, H. B.; Scuseria, G. E.; Robb, M. A.; Cheeseman, J. R.; Scalmani, G.; Barone, V.; Mennucci, B.; Petersson, G. A.; Nakatsuji, H.; Caricato, M.; Li, X.; Hratchian, H. P.; Izmaylov, A. F.; Bloino, J.; Zheng, G.; Sonnenberg, J. L.; Hada, M.; Ehara, M.; Toyota, K.; Fukuda, R.; Hasegawa, J.; Ishida, M.; Nakajima, T.; Honda, Y.; Kitao, O.; Nakai, H.; Vreven, T.;
Montgomery, J. A., Jr.; Peralta, J. E.; Ogliaro, F.; Bearpark, M.; Heyd, J. J.; Brothers, E.; Kudin, K. N.; Staroverov, V. N.; Kobayashi, R.; Normand, J.; Raghavachari, K.; Rendell, A.; Burant, J. C.; Iyengar, S. S.; Tomasi, J.; Cossi, M.; Rega, N.; Millam, J. M.; Klene, M.; Knox, J. E.; Cross, J. B.; Bakken, V.; Adamo, C.; Jaramillo, J.; Gomperts, R.; Stratmann, R. E.; Yazyev, O.; Austin, A. J.; Cammi, R.; Pomelli, C.; Ochterski, J. W.; Martin, R. L.; Morokuma, K.; Zakrzewski, V. G.; Voth, G. A.; Salvador, P.; Dannenberg, J. J.; Dapprich, S.; Daniels, A. D.; Farkas, Â.; Foresman, J. B.; Ortiz, J. V.; Cioslowski, J.; Fox, D. J. Gaussian'09, revision A.03; Gaussian, Inc.: Wallingford, CT, 2009.

(63) Aidas, K.; Angeli, C.; Bak, K. L.; Bakken, V.; Bast, R.; Boman, L.; Christiansen, O.; Cimiraglia, R.; Coriani, S.; Dahle, P.; Dalskov, E. K.; Ekström, U.; Enevoldsen, T.; Eriksen, J. J.; Ettenhuber, P.; Fernández, B.; Ferrighi, L.; Fliegl, H.; Frediani, L.; Hald, K.; Halkier, A.; Hättig, C.; Heiberg, H.; Helgaker, T.; Hennum, A. C.; Hettema, H.; Hjertenæs, E.; Høst, S.; Høyvik, I.-M.; Iozzi, M. F.; Jansík, B.; Jensen, H. J. Aa; Jonsson, D.; Jørgensen, P.; Kauczor, J.; Kirpekar, S.; Kjærgaard, T.; Klopper, W.; Knecht, S.; Kobayashi, R.; Koch, H.; Kongsted, J.; Krapp, A.; Kristensen, K.; Ligabue, A.; Lutnæs, O. B.; Melo, J. I.; Mikkelsen, K. V.; Myhre, R. H.; Neiss, C.; Nielsen, C. B.; Norman, P.; Olsen, J.; Olsen, J. M. H.; Osted, A.; Packer, M. J.; Pawlowski, F.; Pedersen, T. B.; Provasi, P. F.; Reine, S.; Rinkevicius, Z.; Ruden, T. A.; Ruud, K.; Rybkin, V. V.; Sałek, P.; Samson, C. C. M.; de Merás, A. S.; Saue, T.; Sauer, S. P. A.; Schimmelpfennig, B.; Sneskov, K.; Steindal, A. H.; Sylvester-Hvid, K. O.; Taylor, P. R.; Teale, A. M.; Tellgren, E. I.; Tew, D. P.; Thorvaldsen, A. J.; Thøgersen, L.; Vahtras, O.; Watson, M. A.; Wilson, D. J. D.; Ziolkowski, M.; Ågren, H. The Dalton quantum chemistry program system. WIREs Comput. Mol. Sci. 2014, 4, 269-284.

(64) Stanton, J. F.; Gauss, J.; Cheng, L.; Harding, M. E.; Matthews, D. A.; Szalay, P. G. CFOUR, Coupled-Cluster Techniques for Computational Chemistry, a quantum-chemical program package. With contributions from A. A. Auer, R. J. Bartlett, U. Benedikt, C. Berger, D. E. Bernholdt, Y. J. Bomble, O. Christiansen, F. Engel, R. Faber, M. Heckert, O. Heun, M. Hilgenberg, C. Huber, T.-C. Jagau, D. Jonsson, J. Jusélius, T. Kirsch, K. Klein, W. J. Lauderdale, F. Lipparini, T. Metzroth, L. A. Mück, D. P. O’Neill, D. R. Price, E. Prochnow, C. Puzzarini, K. Ruud, F. Schiffmann, W. Schwalbach, C. Simmons, S. Stopkowicz, A. Tajti, J. Vázquez, F. Wang, J. D. Watts and the integral packages MOLECULE (J. Almlöf and P. R. Taylor), PROPS (P. R. Taylor), ABACUS (T. Helgaker, H. J. Aa. Jensen, P. Jørgensen, and J. Olsen), and ECP routines by A. V. Mitin and C. van Wüllen. For the current version, see http://www.cfour.de.

(65) Davidson, E. R.; Feller, D. Basis Set Selection for Molecular Calculations. Chem. Rev. 1986, 86, 681-696.

(66) Maroulis, G. On the bond-length dependence of the static electric polarizability and hyperpolarizability of $\mathrm{F}_{2}$. Chem. Phys. Lett. 2007, 442, 265-269.

(67) Rappoport, D.; Furche, F. Property-optimized Gaussian basis sets for molecular response calculations. J. Chem. Phys. 2010, 133, 134105.

(68) Falden, H. H.; Falster-Hansen, K. R.; Bak, K. L.; Rettrup, S.; Sauer, S. P. A. Benchmarking second order methods for the calculation of vertical electronic excitation energies: Valence and Rydberg states in polycyclic aromatic hydrocarbons. J. Phys. Chem. A 2009, 113, 11995-12012.

(69) Haase, P. A. B.; Faber, R.; Provasi, P. F.; Sauer, S. P. A. Noniterative Doubles Corrections to the Random Phase and Higher Random Phase Approximations: Singlet and Triplet Excitation Energies. J. Comput. Chem. 2020, 41, 43-55.

(70) Kjær, H.; Sauer, S. P. A.; Kongsted, J.; Rusakov, Y. Y.; Krivdin, L. B. Benchmarking SOPPA(CC2) for the calculation of indirect nuclear spin-spin coupling constants: Carbocycles. Chem. Phys. 2011, $381,35-45$.

(71) Hinchliffe, A.; Soscún M., H. J. Ab initio studies of the dipole polarizabilities of conjugated molecules: Part 5 . The five-membered heterocyclic $\mathrm{C}_{4} \mathrm{H}_{4} \mathrm{E}\left(\mathrm{E}=\mathrm{BH}, \mathrm{AlH}, \mathrm{CH}_{2}, \mathrm{SiH}_{2}, \mathrm{NH}, \mathrm{PH}, \mathrm{O}\right.$ and $\left.\mathrm{S}\right) . J$. Mol. Struct.: THEOCHEM 1995, 331, 109-125. 
(72) Kamada, K.; Ueda, M.; Nagao, H.; Tawa, K.; Sugino, T.; Shmizu, Y.; Ohta, K. Molecular Design for Organic Nonlinear Optics: Polarizability and Hyperpolarizabilities of Furan Homologues Investigated by $\mathrm{Ab}$ Initio Molecular Orbital Method. J. Phys. Chem. A 2000, 104, 4723.

(73) Soscún, H.; Bermúdez, Y.; Castellano, O.; Hernández, J. Effects on protonation on the dipole polarizability of monocyclic azines: a theoretical study. Chem. Phys. Lett. 2004, 396, 117-121.

(74) El-Bakali Kassimi, N.; Doerksen, R. J.; Thakkar, A. J. Polarizabilities of Oxazoles: $\mathrm{Ab}$ Initio Calculations and Simple Models. J. Phys. Chem. 1996, 100, 8752-8757.

(75) El-Bakali Kassimi, N.; Lin, Z. Aza-Substituted Thiophene Derivatives: Structures, Dipole Moments, and Polarizabilities. J. Phys. Chem. A 1998, 102, 9906-9911. 